\title{
A New Spectral Element Method for Pricing European Options Under the Black-Scholes and Merton Jump Diffusion Models
}

\author{
Feng Chen · Jie Shen • Haijun Yu
}

Received: 2 June 2011 / Revised: 27 September 2011 / Accepted: 28 October 2011 /

Published online: 11 November 2011

(C) Springer Science+Business Media, LLC (outside the USA) 2011

\begin{abstract}
We present a new spectral element method for solving partial integro-differential equations for pricing European options under the Black-Scholes and Merton jump diffusion models. Our main contributions are: (i) using an optimal set of orthogonal polynomial bases to yield banded linear systems and to achieve spectral accuracy; (ii) using Laguerre functions for the approximations on the semi-infinite domain, to avoid the domain truncation; and (iii) deriving a rigorous proof of stability for the time discretizations of European put options under both the Black-Scholes model and the Merton jump diffusion model. The new method is flexible for handling different boundary conditions and non-smooth initial conditions for various contingent claims. Numerical examples are presented to demonstrate the efficiency and accuracy of the new method.
\end{abstract}

Keywords Spectral element · Spectral-Galerkin · Unbounded domain · Laguerre functions $\cdot$ Option pricing $\cdot$ Black-Scholes $\cdot$ Merton jump diffusion

\section{Introduction and Motivation}

In the classical Black-Scholes model [4], the stock price is a standard Wiener process which is continuous in time. In order to describe phenomena such as unexpected economic turmoils, the jump diffusion model is introduced in [25]. In the jump diffusion model, the

This work is partially supported by NSF DMS-0915066 and AFOSR FA9550-08-1-0416.

F. Chen · J. Shen $(\bowtie)$

Department of Mathematics, Purdue University, West Lafayette, IN 47907-1957, USA

e-mail: shen@math.purdue.edu

F. Chen

e-mail: chen221@math.purdue.edu

H. Yu

LSEC, Institute of Computational Mathematics, Academy of Mathematics and System Sciences, Chinese Academy of Sciences, Beijing 100190, People's Republic of China

e-mail: hyu@1sec.cc.ac.cn 
dynamics of the underline asset is a Lévy process, which, on the one hand, preserves many desirable properties of the Wiener process, such as independent and stationary increments, on the other hand, can capture the feature of volatility smile that is absent in the BlackScholes model. Therefore the jump diffusion model remains one of the most active areas of financial mathematics (c.f., [3, 7, 10, 27, 35, 36, 40]).

Option pricing is an important problem in financial mathematics. The pricing for the European style contingent claims has been extensively studied. Though analytic solutions are most preferred by practitioners (as indicated in [6]), they are not available for many existing financial derivatives on the market. In general, one can write the option price as the conditional expectation of the payoff function and compute it with Monte-Carlo simulations, which is widely used in financial industry. Another popular approach for pricing options, the transform method, was introduced in [8] and unified in [21]. It yields an elegant and fast solution to a wide variety of options. Its another advantage is the immediate extension to the multi-asset cases (c.f., [18, 22]). However, it requires analytic representations of characteristic functions of the stock price, which are not always available.

In this paper we focus on the PDE approach for the option pricing, and attempt to address three of the main difficulties in this approach. First, the infinite generator of the Lévy process is a nonlocal operator with an integral term. Thus a partial integro-differential equation (PIDE) for the option price is derived within the Black-Scholes framework. Second, many payoff functions possess slope discontinuities which might cause unwanted numerical oscillations in numerical solutions of option prices. Third, the PIDE is defined on an unbounded domain, which is often truncated in practice with potential large truncation errors. Therefore an accurate right boundary condition becomes crucial for the numerical computation. This is not a serious issue for the Black-Scholes model, in which the solution decays very fast and the truncated region does not need to be large. However, in some cases such as the Merton jump diffusion model we consider here, the decaying rate is slow, so a large domain with a large number of grid points have to be used with the domain truncation. Another way to get a correct boundary condition with the localization is to use the transparent boundary condition (cf. [1]), which is popular in computational electromagnetics. However, this requires a considerable amount of calculations which affects its flexibility.

For the spatial discretization, the finite difference method and finite element method (c.f., $[2,9,14,35,37])$ have been used extensively in the community of computational finance and has achieved successes in many cases for its effectiveness and flexibility. However, as a low order method, a considerable number of grid points is needed to resolve the solution. Due to the recent thriving of algorithmic trading, the financial market becomes more sensitive to small bid-offer spread. It has at least two significant implications. First, the pricing process should be fast enough to allow a large amount of trading operations within a short time of period (i.e., high frequency trading). Secondly, it is beneficial to have a high order accuracy of the numerical solution. Therefore we consider in this paper a new spectral element method for the option pricing. It possesses both the spectral accuracy of the spectral method and the domain flexibility of the finite element method. Although the spectral element method is widely used in computational sciences and engineerings (cf. [13, 19]), its application to the computational finance is still limited. Its main advantage is that, for a given accuracy, much less grid points, and in many case much less computing times, are needed comparing to low order methods. In fact, to achieve a fixed accuracy, the numerical results presented in Sect. 5 show that our new spectral-element method is more efficient than a usual spectral-element method in [40] and can be orders of magnitude faster than a low-order finite difference method. 
It is well-known that option prices and their derivatives usually change dramatically near slope discontinuities of the payoff functions. By splitting the domain at the slope discontinuities, the solution can be effectively resolved by the spectral element method (c.f., [38-40]). Thus there is no need to artificially smooth the initial data as in [26]. However, previous spectral element implementations use the nodal Lagrange polynomial basis functions which result in linear system with dense block diagonals. In this paper, we use modal bases functions (c.f., $[28,30]$ ) which lead to linear systems with banded block diagonals so it can be efficiently solved. Unlike in previous approach, we treat the unbounded domain directly by using an infinite element, avoiding the domain truncation error. We refer to [30] for the recent survey of spectral methods on unbounded domains, [23] for pricing game options by using Hermite polynomials, and [12] for evaluating bonds by using Laguerre polynomials. Another advantage of having a spectral representation is that the hedging strategies $\Delta$ and $\Gamma$, which are first and second derivatives of the option price [20,34], can be computed directly and accurately from the spectral representation. This is much more efficient and accurate than using a lower order method. Another difficulty in solving the PIDE is how to treat the integral term. Successful methods for implicit treatments include the fixed point iteration [14], the extrapolation [17], solving its associated heat equations [24], etc. We shall treat the integral term in the PIDE explicitly with a Crank-Nicholson-Leap-Frog (CNLF) scheme which we shall prove is stable. Numerical experiments show this approach is very efficient.

The rest of the paper is organized as follows. In Sect. 2 we briefly describe the Lévy driven market model and introduce the PIDE for the European option pricing under the Merton jump diffusion model. In Sect. 3 we describe our time advancing schemes and prove their stabilities. We then present in Sect. 4 our spectral element method together with the treatment of the integral. Numerical results of the European put option in the Black-Scholes model and the Merton jump diffusion model are given in Sect. 5. We end the paper with a few concluding remarks.

\section{Markets Driven by Lévy Processes}

Let $X_{t}$ be a Lévy process defined on a probability space $(\Omega, \mathcal{F}, \mathbb{P})$. Denote $\iota=\sqrt{-1}$. The Lévy-Khintchine representation theorem states that the characteristic function of $X_{t}$ can be written as

$$
\mathbf{E}^{\mathbb{P}}\left[e^{-l z X_{t}}\right]=e^{-t \psi(z)}
$$

with the Lévy-Khintchine exponent

$$
\psi(z)=-\left\{-\frac{\sigma^{2} z^{2}}{2}+\iota \gamma z+\int_{-\infty}^{\infty}\left(e^{\imath z x}-1-\iota z x \chi_{|x| \leq 1}\right) v(\mathrm{~d} x)\right\},
$$

where $\chi_{\Omega}$ stands for the characteristic function for the set $\Omega$. The triplet $(\sigma, \gamma, \nu)$ is known as the generating Lévy triplet with $\sigma>0, \gamma \in \mathbb{R}$, and $v$ being a positive measure satisfying

$$
\int_{-1}^{1} x^{2} v(\mathrm{~d} x)<\infty, \quad \int_{|x|>1} v(\mathrm{~d} x)<\infty .
$$

In the classic Black-Scholes model, $X_{t}$ is a standard Brownian motion, so $v=0$. In the Merton jump diffusion model, $X_{t}$ is the sum of a Brownian motion and a compound Poisson 
process:

$$
X_{t}=\sigma W_{t}+\sum_{i=1}^{N_{t}} U_{i}
$$

where $N_{t}$ is a Poisson process with intensity $\lambda=\int_{-\infty}^{\infty} v(\mathrm{~d} x)<\infty$ and $U_{i}$ 's are independent and identically distributed (i.i.d.) random variables with density $v(\mathrm{~d} x) / \lambda$. In this case, (2.2) is reduced to

$$
\psi(z)=-\left\{-\frac{\sigma^{2} z^{2}}{2}+\iota \gamma_{0} z+\int_{-\infty}^{\infty}\left(e^{\iota z x}-1\right) v(\mathrm{~d} x)\right\} .
$$

Suppose the dynamics of the stock price is given by

$$
S_{t}=S_{0} e^{r t+X_{t}},
$$

where $r$ is the constant interest rate. The infinitesimal generator of the Markovian process $S_{t}$ is an integro-differential operator defined as:

$$
\begin{aligned}
\mathbf{L}^{S_{t}} f(x) & =\lim _{t \rightarrow 0} \frac{\mathbf{E}\left[f\left(x+S_{t}\right)\right]-f(x)}{t} \\
& =\frac{\sigma^{2} x^{2}}{2} f_{x x}-r x f_{x}-r f-\int_{\mathbb{R}}\left(f\left(x e^{y}\right)-f(x)-x\left(e^{y}-1\right) f_{x}\right) v(\mathrm{~d} y) .
\end{aligned}
$$

There exists an equivalent risk-neutral measure, $\mathbb{Q}$, under which $e^{-r t} S_{t}$ becomes a martingale as long as the triplet $(\sigma, \gamma, \nu)$ satisfies the following conditions:

$$
\begin{aligned}
& \int_{|y|>1} v(\mathrm{~d} y) e^{y}<\infty, \\
& \gamma=-\frac{\sigma^{2}}{2}-\int\left(e^{-1}-y \chi_{|y|>1}\right) v(\mathrm{~d} y) .
\end{aligned}
$$

Consider a contingent claim with a deterministic maturity $T$ and payoff $V_{T}$. According to the no-arbitrage principle, its price $V(S, t)$ at a given time $t$ and $S_{t}=S$ is

$$
V(S, t)=\mathbf{E}^{\mathbb{Q}}\left[e^{-r(T-t)} V_{T} \mid S_{t}=S\right] .
$$

By applying Itô's formula to $V\left(S_{t}, t\right)$, we obtain the partial integro-differential equations (PIDE) on an unbounded domain:

$$
V_{t}(S, t)+\mathbf{L}^{S_{t}} V(S, t)=0, \quad S \in(0, \infty), t>0
$$

with the terminal condition

$$
V(S, T)=V_{T} .
$$

Here we restrict the volatility $\sigma(S, T)$ to be non-vanishing. Viscosity solutions of the PIDE are considered in [11] for the pure jump model, i.e., $\sigma(S, T) \equiv 0$. In the case of the European option under the Merton jump diffusion, $U_{i}$ 's in (2.4) are identically, independently and normally distributed. 
With the substitution $v(S, \tau)=V(S, T-\tau)$, we obtain the following Cauchy problem:

$$
v_{\tau}=\frac{\sigma^{2} S^{2}}{2} v_{S S}+(r-\lambda \kappa) S v_{S}-(r+\lambda) v+\lambda \int_{0}^{\infty} v(\eta S, \tau) G(\eta) \mathrm{d} \eta
$$

with the integral kernel

$$
G(\eta)=\frac{1}{\sqrt{2 \pi} \delta \eta} e^{-\frac{(\ln \eta-m)^{2}}{2 \delta^{2}}}
$$

and the initial condition for a put option

$$
v(S, 0)=\max \{K-S, 0\},
$$

where $K$ is the strike price, $m=\mathbb{E} U_{i}$ and $\kappa=\mathbb{E}\left(U_{i}-1\right)$. The payoff function of (2.14) has a slope discontinuity at $S=K$. When $\lambda=0,(2.12)$ reduces to the celebrated Black-Scholes equation:

$$
v_{\tau}=\frac{\sigma^{2} S^{2}}{2} v_{S S}+r S v_{S}-r v, \quad S \in(0,+\infty), \tau>0 .
$$

The hedging strategies are given by

$$
\Delta=v_{S}, \quad \Gamma=v_{S S} .
$$

While it is well-known that the solution of (2.10) goes to zero at the right infinity (cf, [11]), the rate of decay can be very slow in the Merton jump diffusion model. Consequently, when $S$ is large, domain truncation may introduce significant errors. In the example (5.1), a truncation at $S=20 \mathrm{~K}$ with a homogeneous Dirichlet boundary condition will introduce a truncation error in the order of $10^{-3}$ (see Fig. 1). This is the main reason that we deal with the semi-infinite domain directly (see Sect. 4.1). Other kinds of options with different payoff functions and other markets driven by different Lévy processes can also be described by (2.10) and (2.11) with a decaying condition as $S \rightarrow+\infty$. Thus our method maybe used for pricing contingent claims with fixed maturities.

\section{Time Discretizations}

We first rewrite (2.12) in a form which more convenient for numerical approximations:

$$
v_{\tau}=\frac{\sigma^{2}}{2}\left(S^{2} v_{S}\right)_{S}+\left(r-\lambda \kappa-\sigma^{2}\right) S v_{S}-(r+\lambda) v+\lambda \int_{0}^{\infty} v(\eta S, \tau) G(\eta) \mathrm{d} \eta .
$$

We define differential operators

$$
\begin{aligned}
& \mathcal{L}^{\mathrm{BS}} v \triangleq \frac{\sigma^{2}}{2}\left(S^{2} v_{S}\right)_{S}+\left(r-\sigma^{2}\right) S v_{S}-r v, \\
& \mathcal{L}^{\mathrm{M}} v \triangleq \frac{\sigma^{2}}{2}\left(S^{2} v_{S}\right)_{S}+\left(r-\lambda \kappa-\sigma^{2}\right) S v_{S}-(r+\lambda) v,
\end{aligned}
$$

and the integral operator

$$
\mathcal{R} v(S) \triangleq \int_{0}^{\infty} v(\eta S) G(\eta) \mathrm{d} \eta
$$




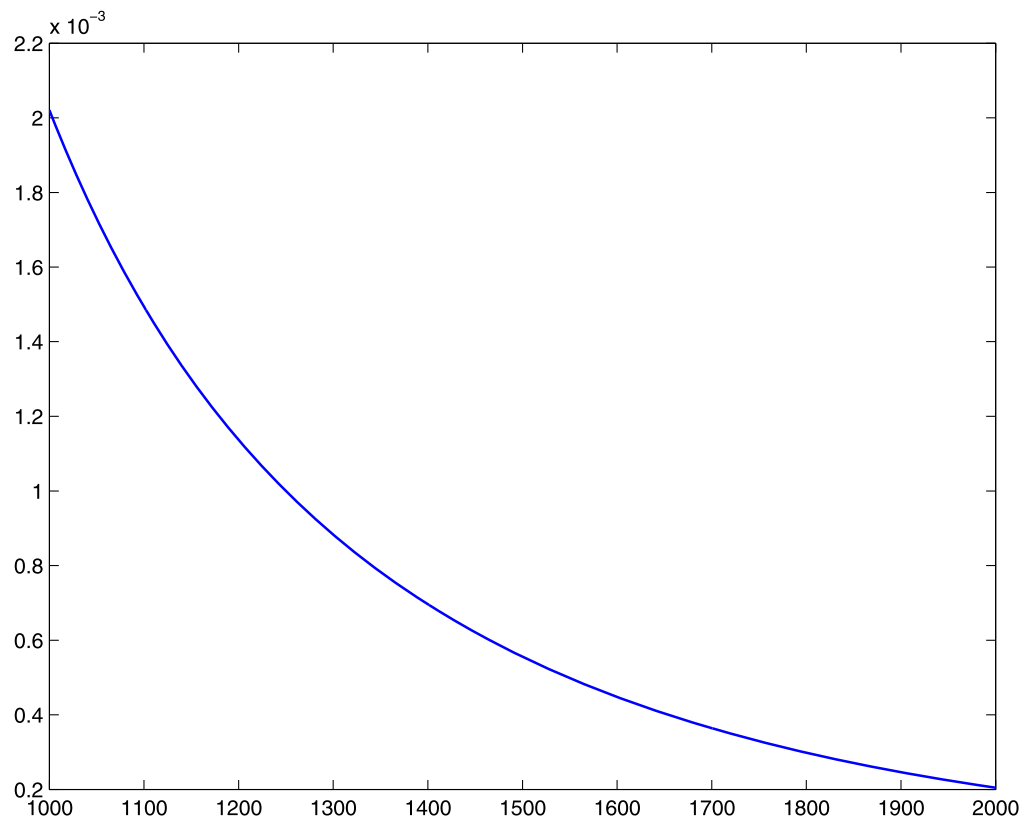

Fig. 1 The European put option under the Merton jump diffusion model with parameters in (5.1); The $x$-axis represents the stock price, and $y$-axis the option price

With a substitution of $\eta S=Z$ in (3.3), we have

$$
\mathcal{R} v(S)=\int_{0}^{\infty} v(Z) \bar{G}(Z, S) \mathrm{d} Z
$$

where

$$
\bar{G}(Z, S) \triangleq \frac{1}{S} G\left(\frac{Z}{S}\right)=\frac{1}{\sqrt{2 \pi} \delta Z} e^{-\frac{\left(\ln \frac{Z}{S}-m\right)^{2}}{2 \delta^{2}}} .
$$

With the above notations, the Black-Scholes equation, i.e., (3.1) with $\lambda=0$, becomes

$$
v_{t}=\mathcal{L}^{\mathrm{BS}} v,
$$

and the PIDE (3.1) becomes

$$
v_{t}=\mathcal{L}^{\mathrm{M}} v+\mathcal{R} v .
$$

Consider the second order Crank-Nicholson scheme for (3.6):

$$
\frac{v^{k+1}-v^{k}}{\delta t}=\frac{\mathcal{L}^{\mathrm{BS}} v^{k}+\mathcal{L}^{\mathrm{BS}} v^{k+1}}{2} .
$$

At each time step, one needs to solve the following model problem, a linear elliptic equation with variable coefficients:

$$
\left\{\begin{array}{l}
-c_{1}\left(x^{2} v^{\prime}(x)\right)^{\prime}+c_{2} x v^{\prime}(x)+c_{3} v(x)=f(x), \quad x \in \Omega=(0,+\infty), \\
\lim _{x \rightarrow \infty} v(x)=0
\end{array}\right.
$$


where $c_{1}, c_{2}$, and $c_{3}$ are constants depending on $\sigma, r$, and $\delta t$. Note that the system (3.9) does not need any left boundary condition to guarantee its well-posedness due to the degenerate nature of $x^{2}$ in the leading term.

Theorem 3.1 The scheme (3.8) is unconditionally stable. More precisely, we have for all $k \delta t \leq T$,

$$
\begin{cases}\left\|v^{k+1}\right\| \leq\left\|v^{k}\right\|, & \text { if } \alpha \leq 0 \\ \left\|v^{k}\right\|^{2} \leq C e^{2 \alpha T}\left\|v^{0}\right\|^{2}, & \text { if } \alpha>0\end{cases}
$$

where $\alpha \triangleq \frac{\left(r-\sigma^{2}\right)^{2}-2 r \sigma^{2}}{4 \sigma^{2}}$.

Proof Taking the inner product of (3.8) with $v^{k+1}+v^{k}$, we derive

$$
\begin{aligned}
\frac{\left\|v^{k+1}\right\|^{2}-\left\|v^{k}\right\|^{2}}{\delta t}= & -\frac{\sigma^{2}}{2} \frac{\left\|S\left(v_{S}^{k+1}+v_{S}^{k}\right)\right\|^{2}}{2} \\
& +\left(r-\sigma^{2}\right) \frac{\left(S\left(v_{S}^{k+1}+v_{S}^{k}\right), v^{k+1}+v^{k}\right)}{2}-r \frac{\left\|v^{k+1}+v^{k}\right\|^{2}}{2} .
\end{aligned}
$$

A simple manipulation leads to

$$
\frac{\left\|v^{k+1}\right\|^{2}-\left\|v^{k}\right\|^{2}}{\delta t}=-\frac{\sigma^{2}}{4} P^{k}+\alpha\left\|v^{k+1}+v^{k}\right\|^{2},
$$

where

$$
P^{k}=\left\|S\left(v_{S}^{k+1}+v_{S}^{k}\right)-\frac{r-\sigma^{2}}{\sigma^{2}}\left(v^{k+1}+v^{k}\right)\right\|^{2} .
$$

Therefore, we have $\left\|v^{k+1}\right\| \leq\left\|v^{k}\right\|$, if $\alpha \leq 0$. When $\alpha>0$, we apply the triangle inequality on $\left\|v^{k+1}+v^{k}\right\|^{2}$ in (3.12) to get

$$
\frac{\left\|v^{k+1}\right\|^{2}-\left\|v^{k}\right\|^{2}}{\delta t} \leq 2 \alpha\left(\left\|v^{k+1}\right\|^{2}+\left\|v^{k}\right\|^{2}\right)
$$

which implies

$$
\left\|v^{k+1}\right\|^{2} \leq \frac{1+2 \alpha \delta t}{1-2 \alpha \delta t}\left\|v^{k}\right\|^{2} \leq\left(\frac{1+2 \alpha \delta t}{1-2 \alpha \delta t}\right)^{k+1}\left\|v^{0}\right\|^{2} \leq C e^{2 \alpha T}\left\|v^{0}\right\|^{2} .
$$

Next we consider the Crank-Nicholson Leap-Frog scheme for (3.7):

$$
\frac{v^{k+1}-v^{k-1}}{2 \delta t}=\frac{\mathcal{L}^{\mathrm{M}} v^{k+1}+\mathcal{L}^{\mathrm{M}} v^{k-1}}{2}+\lambda \mathcal{R} v^{k}, \quad \forall k \geq 1,
$$

with

$$
\frac{v^{1}-v^{0}}{\delta t}=\mathcal{L}^{\mathrm{M}} v^{1}+\lambda \mathcal{R} v^{0}
$$

Note that the form of (3.4) is suitable for the numerical implementation since the integral term is treated explicitly in the time discretization (see Sect. 4.2). 
In order to prove the stability for the scheme (3.16), we need the following lemma (c.f., [16]).

Lemma 3.2 (Heat kernel) Consider the one dimensional heat equation:

$$
\left\{\begin{array}{l}
u_{t}=u_{x x}, \quad(x, t) \in \mathbb{R} \times(0, T] \\
u(x, 0)=g(x) \\
\lim _{x \rightarrow \pm \infty} u(x)=0 .
\end{array}\right.
$$

Assume that $g$ is bounded, continuous, nonnegative, and non-vanishing, then

$$
u(x, t)=\frac{1}{\sqrt{2 \pi} \sqrt{2 t}} \int_{-\infty}^{+\infty} \exp \left[-\frac{(x-y)^{2}}{2(\sqrt{2 t})^{2}}\right] g(y) \mathrm{d} y .
$$

Without loss of generality, we set $m$ in (2.13) to be zero in the following analysis. When $m \neq 0$, one can shift the coordinate accordingly to get similar results. Next lemma provides the exact solution to a one dimensional advection-diffusion problem and establishes its connection with the integral kernel of our problem.

Lemma 3.3 (Advection-diffusion) Assume $m=0$, then $\mathcal{R} v(S)=w\left(S, \frac{\delta^{2}}{2}\right)$, where $w(S, t)$ is the solution to the following one dimensional advection-diffusion equation:

$$
\left\{\begin{array}{l}
w_{t}=S^{2} w_{S S}+S w_{S}, \quad(S, t) \in \mathbb{R}^{+} \times(0, T] \\
w(S, 0)=v(S) \\
\lim _{S \rightarrow \infty} w(S, t)=0 .
\end{array}\right.
$$

Proof Consider the coordinate transformation

$$
x=\ln S, \quad S=e^{x} .
$$

Define $u(x) \triangleq w(S(x))=w\left(e^{x}\right)$, then

$$
u_{t}=w_{t}, \quad u_{x}=S w_{S}, \quad u_{x x}=S w_{S}+S^{2} w_{S S} .
$$

So the system (3.20) is transformed into

$$
\left\{\begin{array}{l}
u_{t}=u_{x x}, \quad(x, t) \in \mathbb{R} \times(0, T], \\
u(x, 0)=v\left(e^{x}\right), \\
\lim _{x \rightarrow \pm \infty} u(x)=0 .
\end{array}\right.
$$

According to Lemma 3.2, we have

$$
u(x, t)=\frac{1}{\sqrt{2 \pi} \sqrt{2 t}} \int_{-\infty}^{+\infty} \exp \left[-\frac{(x-y)^{2}}{2(\sqrt{2 t})^{2}}\right] v\left(e^{y}\right) \mathrm{d} y .
$$

Next we transform the coordinate back:

$$
S=e^{x}, \quad Z=e^{y} .
$$


The solution in (3.24) becomes

$$
w(S, t)=\frac{1}{\sqrt{2 \pi} \sqrt{2 t}} \int_{0}^{+\infty} \exp \left[-\frac{\left(\ln \frac{S}{Z}\right)^{2}}{2(\sqrt{2 t})^{2}}\right] \frac{v(Z)}{Z} \mathrm{~d} Z .
$$

Therefore $\mathcal{R} v(S)=w\left(S, \frac{\delta^{2}}{2}\right)$ by comparing (3.26) with (3.4).

Lemma $3.4\left(L^{2}\right.$-bound) $\|\mathcal{R} v(S)\|^{2} \leq C(\delta)\|v(S)\|^{2}$, where $C(\delta)=e^{\frac{\delta^{2}}{2}}$.

Proof According to Lemma 3.3, it is sufficient to estimate $w\left(S, \frac{\delta^{2}}{2}\right)$. Taking the inner product of (3.20) with $w$, we have

$$
\frac{1}{2} \frac{\mathrm{d}}{\mathrm{d} t}\|w\|^{2}=-\left\|S w_{S}\right\|^{2}-\left(S w_{S}, w\right)
$$

In addition,

$$
\left(S w_{S}, w\right)=\frac{1}{2}\left(S,\left(w^{2}\right)_{S}\right)=\frac{1}{2}\left(\left.S w^{2}\right|_{S=0} ^{S=+\infty}-\|w\|^{2}\right)=-\frac{1}{2}\|w\|^{2} .
$$

Combining (3.27) and (3.28), we get

$$
\frac{\mathrm{d}}{\mathrm{d} t}\|w\|^{2} \leq\|w\|^{2}
$$

By using the Gronwall's inequality on $\left[0, \frac{\delta^{2}}{2}\right]$, we derive

$$
\|\mathcal{R} v(S)\|^{2}=\left\|w\left(S, \frac{\delta^{2}}{2}\right)\right\|^{2} \leq e^{\frac{\delta^{2}}{2}}\|w(S, 0)\| .
$$

Theorem 3.5 ( $L^{2}$-stability) There exists a constant $\beta$ depending on $\alpha, \lambda$ and $\delta$ such that for all $\delta t<\frac{1}{\beta}$, we have

$$
\left\|v^{m}\right\|^{2} \leq C\left\|v^{0}\right\|^{2}, \quad \forall 2 \leq m \leq \frac{T}{\delta t}-1,
$$

where $C$ is a generic constant depending on $\alpha, \lambda, \delta$ and $T$.

Proof Taking inner product of (3.16) with $v^{k+1}+v^{k-1}$, we have

$$
\frac{\left\|v^{k+1}\right\|^{2}-\left\|v^{k-1}\right\|^{2}}{2 \delta t}=-\frac{\sigma^{2}}{4} P^{k}+\alpha\left\|v^{k+1}+v^{k-1}\right\|^{2}+\lambda\left(\mathcal{R} v^{k}, v^{k+1}+v^{k-1}\right),
$$

where

$$
P^{k}=\left\|S\left(v_{S}^{k+1}+v_{S}^{k-1}\right)-\frac{r-\sigma^{2}}{\sigma^{2}}\left(v^{k+1}+v^{k-1}\right)\right\|^{2} .
$$


Applying the triangle inequality and Lemma 3.4, we derive

$$
\begin{aligned}
\frac{\left\|v^{k+1}\right\|^{2}-\left\|v^{k-1}\right\|^{2}}{2 \delta t} \leq & 2 \alpha\left(\left\|v^{k+1}\right\|^{2}+\left\|v^{k-1}\right\|^{2}\right) \\
& +\frac{\lambda}{2}\left(C(\delta)\left\|v^{k}\right\|^{2}+2\left\|v^{k+1}\right\|^{2}+2\left\|v^{k-1}\right\|^{2}\right) \\
\leq & \frac{1}{2}\left(\beta_{1}\left\|v^{k+1}\right\|^{2}+\beta_{0}\left\|v^{k}\right\|^{2}+\beta_{-1}\left\|v^{k-1}\right\|^{2}\right) \\
\leq & \frac{\beta}{6}\left(\left\|v^{k+1}\right\|^{2}+\left\|v^{k}\right\|^{2}+\left\|v^{k-1}\right\|^{2}\right),
\end{aligned}
$$

where

$$
\beta_{1}=\beta_{-1}=4 \alpha+2 \lambda, \quad \beta_{0}=C(\delta) \lambda, \quad \frac{\beta}{3}=\max \left\{\beta_{1}, \beta_{0}, \beta_{-1}\right\} .
$$

Without loss of generality, we assume $m$ to be an even number. Summing up (3.34) for odd $k$ between 1 and $m-1$, we derive

$$
\begin{aligned}
\frac{\left\|v^{m}\right\|^{2}-\left\|v^{0}\right\|^{2}}{\delta t} & \leq \frac{\beta}{3}\left(\sum_{\substack{k=2, k \text { odd }}}^{m}\left\|v^{k}\right\|^{2}+\sum_{\substack{k=1, k \text { odd }}}^{m-1}\left\|v^{k}\right\|^{2}+\sum_{\substack{k=0, k \text { odd }}}^{m-2}\left\|v^{k}\right\|^{2}\right) \\
& \leq \frac{\beta}{3}\left(\sum_{k=2}^{m}\left\|v^{k}\right\|^{2}+\sum_{k=1}^{m-1}\left\|v^{k}\right\|^{2}+\sum_{k=0}^{m-2}\left\|v^{k}\right\|^{2}\right) \\
& \leq \beta \sum_{k=0}^{m}\left\|v^{k}\right\|^{2} .
\end{aligned}
$$

Rearranging terms in (3.36), we get

$$
\left\|v^{m}\right\|^{2} \leq \delta t \beta \sum_{k=0}^{m}\left\|v^{k}\right\|^{2}+\left\|v^{0}\right\|^{2} .
$$

We can then conclude (3.31) by applying the discrete Gronwall's inequality (see, for instance, Lemma B.10 in [32]) to the above.

\section{Spatial Discretizations}

We describe in this section our spectral element method for (3.9) with special treatments for the integral term.

\subsection{The Spectral Element Method}

Consider the following function space:

$$
H_{B}^{1}=\left\{u \in H^{1}(0, \infty): \lim _{x \rightarrow \infty} u(x)=0\right\} .
$$

The week formulation of (3.9) is: Find $u \in H_{B}^{1}$ such that

$$
c_{1}\left(x u^{\prime}, x v^{\prime}\right)+c_{2}\left(x u^{\prime}, v\right)+c_{3}(u, v)=(f, v), \quad \forall v \in H_{B}^{1} .
$$


Next we split the domain $\Omega=(0, \infty)$ into $M$ non-overlapping elements:

$$
\Omega^{1}=\left(x_{0}, x_{1}\right), \ldots, \Omega^{i}=\left(x_{i-1}, x_{i}\right), \ldots, \Omega^{M}=\left(x_{M-1}, x_{M}\right),
$$

where $0=x_{0}<x_{1}<\cdots<x_{M}=\infty$. We define the approximation space

$$
\mathbb{X}_{N}=\left\{u \in C(\Omega) ;\left.u\right|_{\Omega^{i}} \in \mathbb{P}_{n_{i}}, 1 \leq i \leq M-1 ;\left.u\right|_{\Omega^{M}} \in \hat{\mathbb{P}}_{n_{M}}\right\},
$$

where $N$ stands for the dimension of $\mathbb{X}_{N}, \mathbb{P}_{k}$ is the $k$-th degree polynomial space, and $\hat{\mathbb{P}}_{k}=$ $e^{-x / 2} \mathbb{P}_{k}$.

Since the initial condition has a slope discontinuity at $S=K$, it is important that $K$ is one of the nodes $\left\{x_{i}\right\}_{i=1}^{M-1}$ so that the solution can be well approximated in the approximation space $X_{N}$.

The spectral element method for (3.9) is: Find $u_{N}$ such that

$$
\left\langle\mathcal{A} u_{N}, v_{N}\right\rangle:=\left(x u_{N}^{\prime}, x v_{N}^{\prime}\right)+\left(x u_{N}^{\prime}, v_{N}\right)+\left(u_{N}, v_{N}\right)=\left(\mathcal{I}_{N} f, v_{N}\right), \quad \forall v_{N} \in \mathbb{X}_{N},
$$

where $I_{N}: C(\Omega) \rightarrow \mathbb{X}_{N}$ is an interpolation operator based on Legendre-Gauss-Lobatto points at all subintervals $\Omega_{i}$.

We now construct a set of basis functions for $\mathbb{X}_{N}$. We start with the interior modal basis functions $\left\{\phi_{j}^{i}(x)\right\}_{0 \leq j \leq n_{i}-2}^{\substack{1 \leq i \leq M-1 \\ 0 \leq j \leq}}$ for $\Omega_{i}, 1 \leq i \leq M-1$.

$$
\phi_{j}^{i}(x)= \begin{cases}L_{j}^{i}(x)-L_{j+2}^{i}(x), & x \in \Omega^{i}, \\ 0, & \text { else }\end{cases}
$$

where $L_{j}^{i}$ 's are Legendre polynomials defined on the element $\Omega^{i}$, and $n_{i}$ 's are numbers of Gaussian points assigned on $\Omega^{i}$. For the unbounded domain $\Omega^{M}$, the interior modal basis functions are

$$
\phi_{k}=\widehat{\mathcal{L}}_{k}-\widehat{\mathcal{L}}_{k+1},
$$

where $\left\{\widehat{\mathcal{L}}_{k}\right\}_{0 \leq k \leq n_{M-1}}$ are Laguerre functions scaled on $\Omega^{M}$.

The nodal basis functions (see Fig. 2) at $\left\{x_{i}\right\}_{1 \leq i \leq M-2}$ are

$$
I^{i}= \begin{cases}\frac{x_{i+1}-x}{2 d_{i+1}}, & x \in \Omega^{i}, \\ \frac{x-x_{i-1}}{2 d_{i}}, & x \in \Omega^{i-1}, \\ 0, & \text { otherwise, }\end{cases}
$$

with $d_{i}=\frac{x_{i}-x_{i-1}}{2}$. Finally, the nodal basis functions at $x_{0}$ and $x_{M-1}$ are given by

$$
I^{0}= \begin{cases}\frac{x_{1}-x}{2 d_{1}}, & x \in \Omega^{1}, \\ 0, & \text { otherwise }\end{cases}
$$

and

$$
I^{M-1}= \begin{cases}e^{-\frac{x-x_{M}-1}{2}}, & x \in \Omega^{M}, \\ \frac{x-x_{M-1}}{2 d_{M-1}}, & x \in \Omega^{M-1}, \\ 0, & \text { otherwise. }\end{cases}
$$




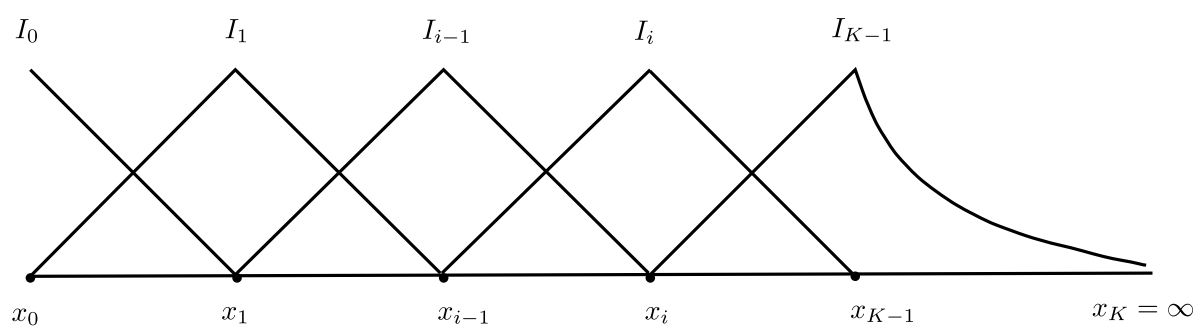

Fig. 2 Nodal functions defined on an unbounded domain
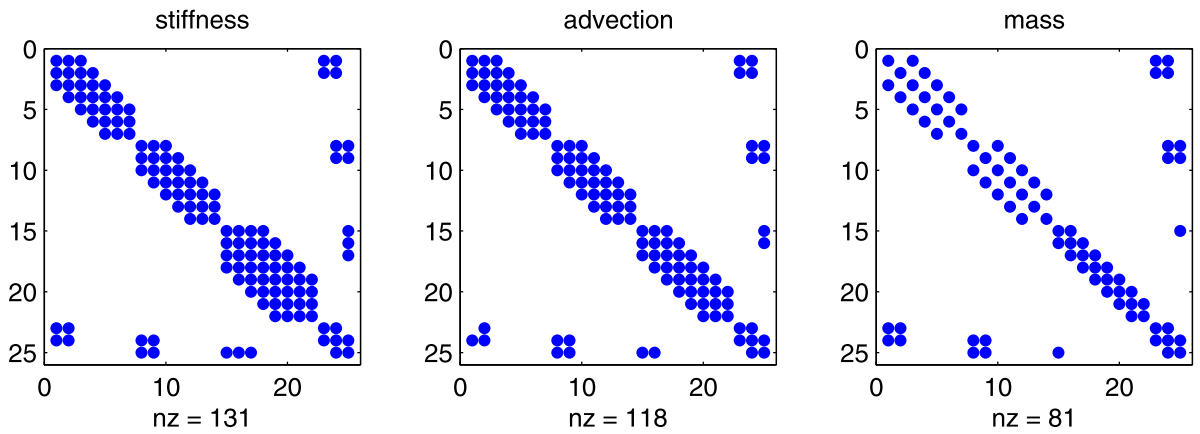

Fig. 3 With 3 elements and 8 Gaussian points in each element

It is easy to verify that the above basis functions form a basis for $\mathbb{X}_{N}$ with the number of degrees of freedom $N=\sum_{i=1}^{M}\left(n_{i}-1\right)+(M-1)$.

Plugging in

$$
u_{N}=\sum_{i=1}^{M} \sum_{j=0}^{n_{i}-2} u_{k i}^{1} \phi_{j}^{i}+\sum_{i=1}^{M-1} u_{i}^{2} I^{i}
$$

in (4.5) and running $v_{N}$ through all basis functions of $\mathbb{X}_{N}$, (4.5) reduces to the following linear system

$$
\left[\begin{array}{ll}
A & B_{1} \\
B_{2} & C
\end{array}\right]\left[\begin{array}{l}
\bar{u}_{1} \\
\bar{u}_{2}
\end{array}\right]=\left[\begin{array}{l}
\bar{f}_{1} \\
\bar{f}_{2}
\end{array}\right],
$$

where with $\bar{u}_{1}$ (resp. $\bar{u}_{2}$ ) is the vector consisting the coefficients of $\left\{u_{k i}^{1}\right\}$ (resp. $\left\{u_{i}^{2}\right\}$ ) in (4.11), $\bar{f}_{1}$ (resp. $\bar{f}_{2}$ ) is the vector consisting of $\left\langle f, \phi_{j}^{1}\right\rangle$ (resp. $\left\langle f, I^{i}\right\rangle$ ), and

$$
A=\operatorname{diag}\left(A^{1}, A^{2}, \ldots, A^{M}\right)
$$

with

$$
A_{k j}^{i}=\left\langle\phi_{j}^{i}, \phi_{k}^{i}\right\rangle, \quad C_{k j}=\left\langle\mathcal{I}_{j}, I_{k}\right\rangle,
$$

$B_{1}$ and $B_{2}$ are rectangular matrices with entries $\left\langle\mathcal{A} \phi_{j}^{i}, I_{k}\right\rangle$ and $\left\langle\mathcal{A} I_{j}, \phi_{k}^{i}\right\rangle$, respectively. Thanks to the properties of Legendre polynomial and Laguerre functions, the matrices $\left\{A^{i}\right\}$, $C, B_{1}$ and $B_{2}$ are sparsely banded (cf. Fig. 3). The linear system (4.12) can be efficiently 
solved by using a block Gaussian elimination as follows:

$$
\left[\begin{array}{cc}
I & A^{-1} B_{1} \\
0 & C-B_{2}^{T} A^{-1} B_{1}
\end{array}\right]\left[\begin{array}{l}
\bar{u}_{1} \\
\bar{u}_{2}
\end{array}\right]=\left[\begin{array}{c}
A^{-1} \bar{f}_{1} \\
f_{2}-B_{2}^{T} A^{-1} \bar{f}_{1}
\end{array}\right] .
$$

Therefore, the computational complexity of our method is dominated by the transforms between physical values and spectral representation with $O\left(\sum_{k=1}^{M} n_{k}^{2}\right)$ flops. This cost can be reduced to $O\left(\sum_{k=1}^{M} n_{k} \log n_{k}\right)$ (with a much large constant) if we use a Chebyshev-Legendre approach on each subdomain (cf. [15, 29, 31]).

\subsection{Discretization of the Integral Term}

Next we deal with the integral term in (2.12). Consider first a direct approximation of $\mathcal{R} v(S)$ by using Legendre-Gauss-Lobatto quadratures on $\left\{\Omega_{i}\right\}_{i=1}^{M-1}$ and Laguerre-GaussRadau quadrature on $\Omega_{M}$ :

$$
\mathcal{R} v(S)=\sum_{i=1}^{M} \int_{\Omega^{i}} v(Z) \bar{G}(Z, S) \mathrm{d} Z \approx \sum_{i=1}^{M} J_{i} \sum_{j=0}^{n_{i}} \omega_{j}^{i} \bar{G}\left(Z\left(\xi_{j}^{i}\right), S\right) v\left(Z\left(\xi_{j}^{i}\right)\right),
$$

where $J_{i}$ is the Jacobian of the affine mapping which maps $\Omega_{i}$ to $(-1,1)$ or $(0, \infty), w_{j}^{i}$ are the quadrature weights, and $Z\left(\xi_{j}^{i}\right)$ are the mapped quadrature points on $\Omega^{i}$.

Note that $\bar{G}(Z, S)$ as a function of $Z$ (see Fig. 4) has a peak of magnitude $O\left(S^{-1}\right)$ in the interval $(0, S]$. In other words, there is a large gradient drop when $S$ is small. Hence, a large number of quadrature points, much larger than what is needed to accurately represent the approximation solution, is needed in $\Omega_{1}$ to achieve the required accuracy. A simple fix for this difficulty is to use the over-integration technique proposed in [40]. Namely, we use $n_{1}$ points to represent $u_{N}$ in $\Omega_{1}$ but $n_{1}^{*} \gg n_{1}$ points to over-integrate the integral term in $\Omega_{1}$.

\section{Numerical Results}

We present in this section some numerical results for European put options under both the Black-Scholes model and the Merton jump diffusion model. The corresponding call option price can be calculated by using the put-call parity. We set the following benchmark parameters throughout all the implementations:

$$
\begin{array}{ll}
T=0.25, & K=100, \quad r=0.05, \quad \sigma=0.15, \\
\lambda=0.1, \quad \delta=0.45, \quad m=-0.9 . &
\end{array}
$$

We recall that an analytic solution to the PIDE (2.12) is:

$$
v=\sum_{n=0}^{\infty} \frac{e^{-\lambda^{\prime} T}\left(\lambda^{\prime} T\right)^{n}}{n !} f_{n}
$$

where $\lambda^{\prime}=\lambda(1+\kappa), \kappa=e^{m+\frac{1}{2} \delta^{2}}-1, \gamma=\ln (1+\kappa)$, and $f_{n}$ is the Black-Scholes option price with instantaneous variance $\sigma^{2}+n \delta^{2} / T$ and risk-free rate $r-\lambda \kappa+n \gamma / T$. We will use (5.2) to validate our numerical methods. 

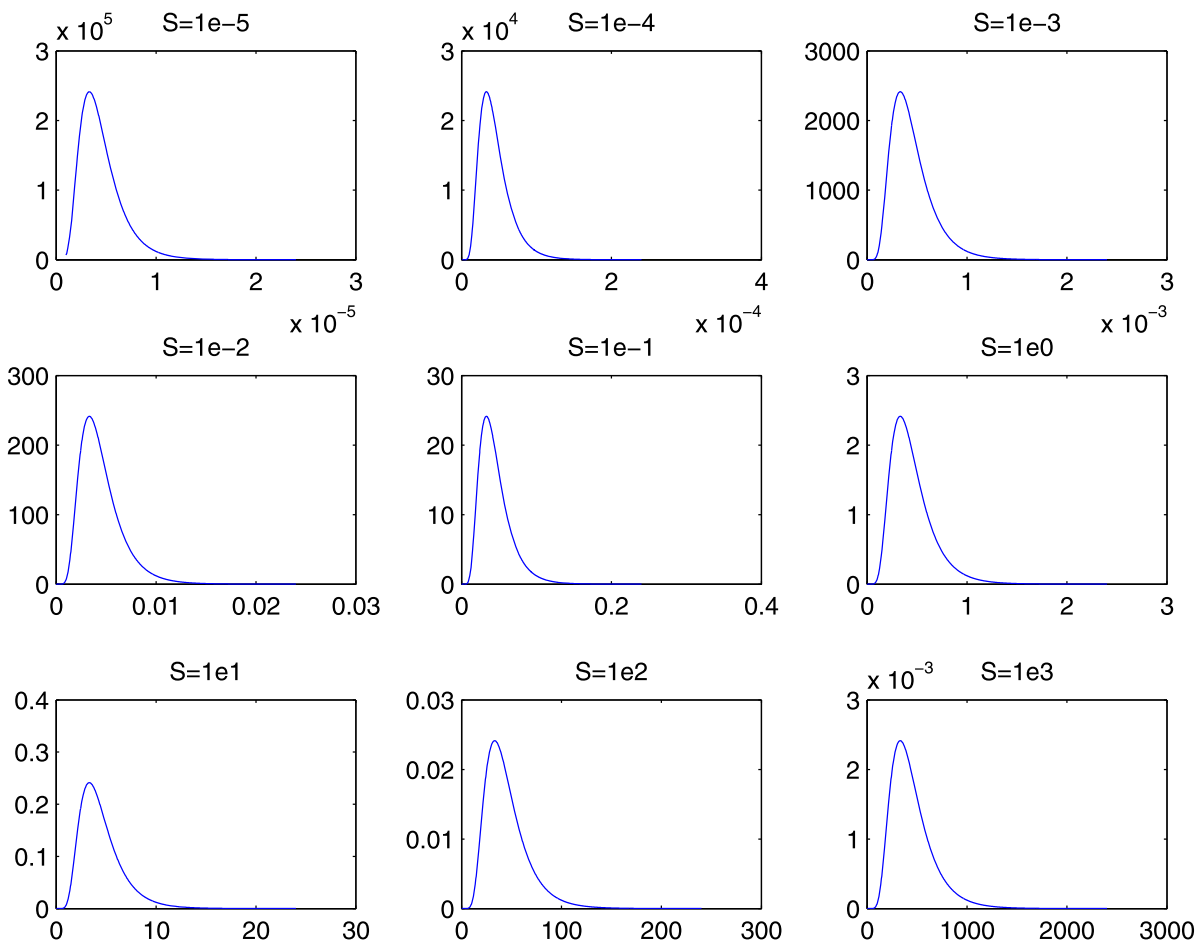

Fig. $4 \bar{G}(Z, S)$ as a function of $Z$ for different $S$

\subsection{Black-Scholes Model}

We use the scheme (3.8) with three elements for the spatial discretization:

$$
\Omega^{1}=(0, K), \quad \Omega^{2}=(K, 2 K), \quad \Omega^{3}=(2 K, \infty) .
$$

Figure 5 shows the agreement between the numerical solution and the theoretical one with $\delta t=10^{-3}$ and the following number of quadrature points in each element:

$$
n_{1}=20, \quad n_{2}=20, \quad n_{3}=6 .
$$

As is well-known (cf. [5]), when using a spectral method for unbounded domains, a proper scaling parameter can often significantly increase the efficiency. In the case of Laguerre functions for $\Omega_{3}$, we can choose the scaling parameter as follows. Assuming that the solution is essentially zero for $S>2 K+\tilde{\xi}$. Then, if $2 K+\xi$ is the largest Laguerre-Gauss-Radau point, we set the scaling parameter to be $\alpha=\xi / \tilde{\xi}$. In this case, the corresponding scaling parameters for $\Omega^{3}$ are:

$$
\tilde{\xi}=K=100, \quad \xi \approx 17.65, \quad \alpha \approx 0.18
$$

Next we examine the temporal and spatial accuracy. We first fix $n_{i}$ in (5.4) and change the time step $\delta t$ gradually. We plot in Fig. 6 the error calculated at the strike which is the point 

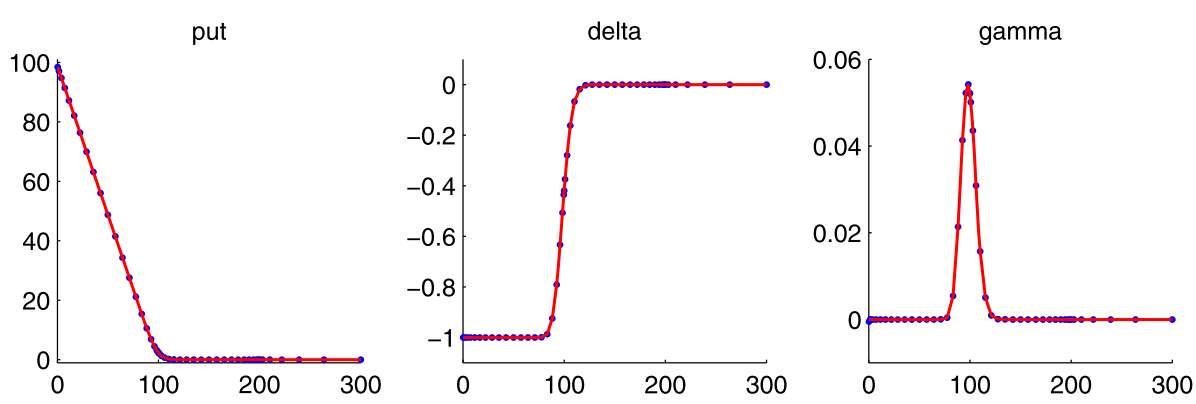

Fig. 5 Black-Scholes model. Line: exact; dotted: numerical
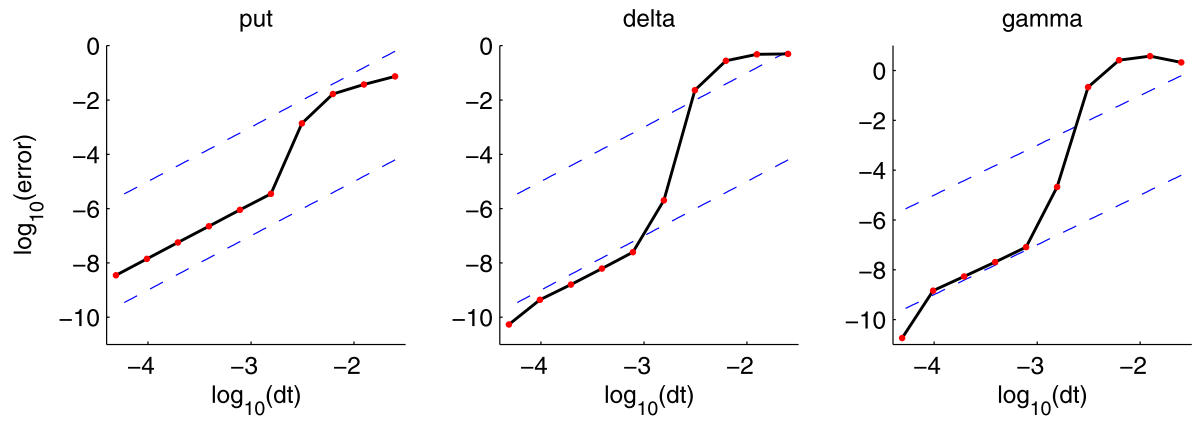

Fig. 6 Black-Scholes model: errors at the strike, with different time step sizes and fixed $\left\{n_{i}\right\}$ in $(5.4)$
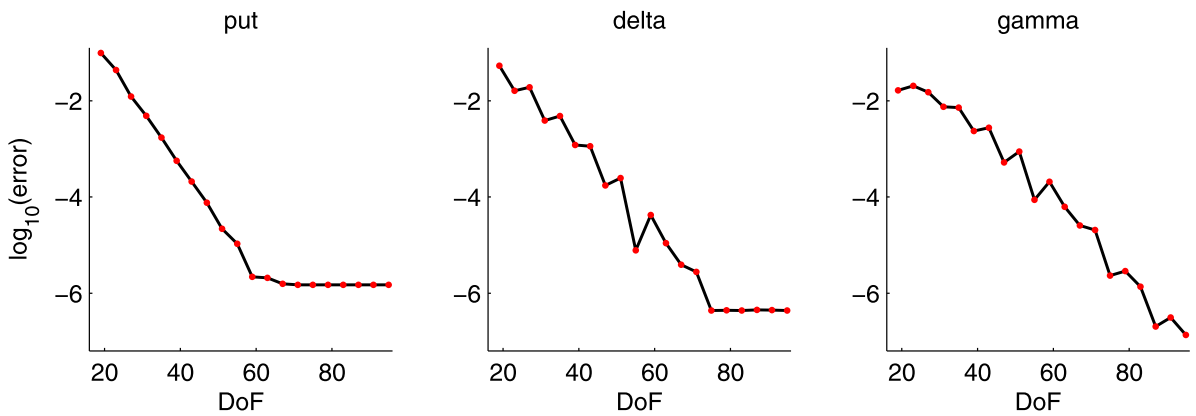

Fig. 7 Black-Scholes model: $L^{\infty}$-errors, with different $\left\{n_{i}\right\}$ and a fixed $\delta t$ of $10^{-3}$

of most financial interest. The dotted lines in Fig. 6 have the slope of 2 which indicates a second order convergence in time. Next, we fix $\delta t=10^{-3}$ and plot in Fig. 7 the errors with different numbers of degrees of freedom (DoF). We observe an exponential convergence in space. 

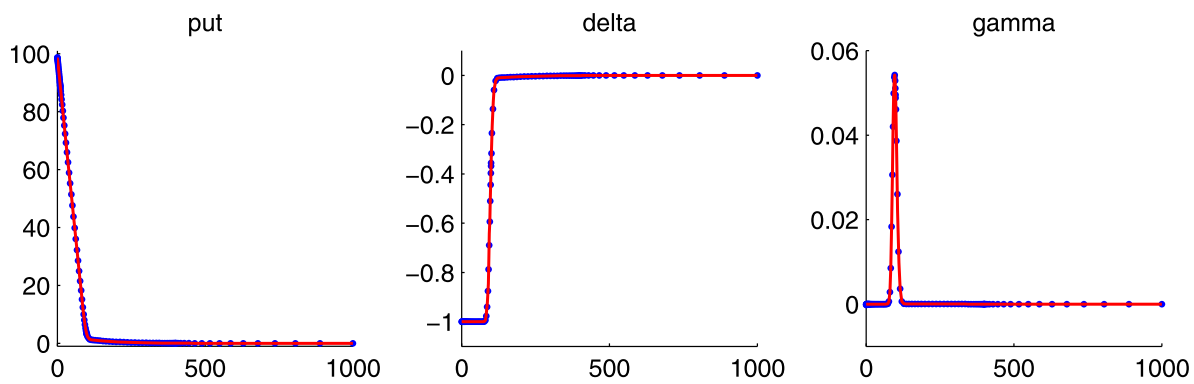

Fig. 8 Merton jump diffusion model. Line: exact; dotted: numerical
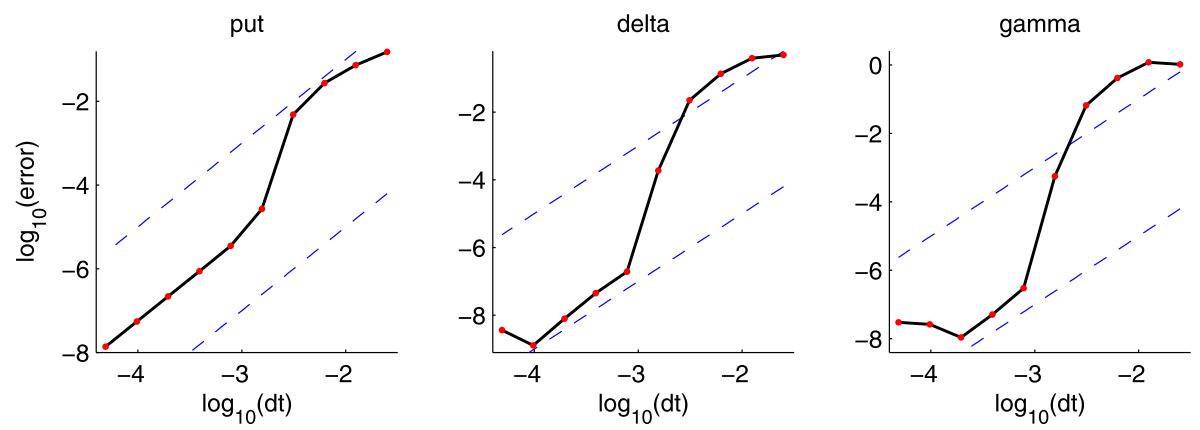

Fig. 9 Merton jump diffusion model: errors at the strike, with different time step sizes and the fixed $\left\{n_{i}\right\}$ in (5.7)

\subsection{Merton Jump Diffusion Model}

We now consider the scheme of (3.16) and (3.17) for the Merton jump diffusion model. For the spatial discretization, we use the following four elements:

$$
\Omega^{1}=(0, \epsilon K), \quad \Omega^{2}=(\epsilon K, K), \quad \Omega^{3}=(K, 4 K), \quad \Omega^{4}=(4 K, \infty) .
$$

In Fig. 8, we plot the numerical and exact solutions with the following computational parameters:

$$
\delta t=10^{-3}, \quad n_{1}=4, \quad n_{2}=n_{3}=36, \quad n_{4}=6, \quad \epsilon=0.1 .
$$

The corresponding scaling parameters for $\Omega^{4}$ are

$$
\alpha \approx 0.09, \quad \xi \approx 53.53, \quad \tilde{\xi}=10 \mathrm{~K} .
$$

In Fig. 9, we plot the errors with fixed $\left\{n_{i}\right\}$ in (5.7) and different $\delta t$, while in Fig. 10, we plot the errors with fixed $\delta t=10^{-3}$. Again a second order convergence in time and exponential convergence in space are observed. Note that the saturation of the error when $\delta t$ is very small in Fig. 9 is due to the spatial discretization as more spatial points are needed to achieve better accuracy for computing $\Delta$ and $\Gamma$, while the saturation of error with larger DoF in Fig. 10 is due to the time discretization error for fixed $\delta t$. 

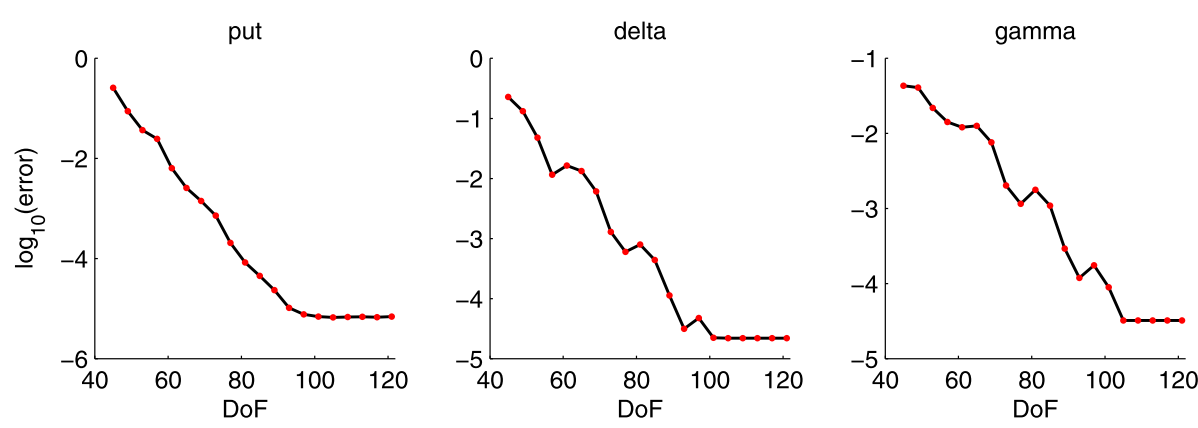

Fig. 10 Merton jump diffusion model: $L^{\infty}$-errors, with different $\left\{n_{i}\right\}$ and a fixed $\delta t$ of $10^{-3}$

Table 1 Computing times for the Merton jump diffusion model

\begin{tabular}{llll}
\hline Computing time (ms.) & $L_{w}^{2}$-error & No. of time steps & No. of spatial points \\
\hline 0.6 & $2.40 \mathrm{e}-3$ & 40 & $35=16 \times 2+3$ \\
1.7 & $7.67 \mathrm{e}-4$ & 80 & $45=20 \times 2+5$ \\
4.3 & $4.50 \mathrm{e}-5$ & 160 & $58=26 \times 2+6$ \\
11.7 & $4.25 \mathrm{e}-6$ & 320 & $72=34 \times 2+6$ \\
\hline
\end{tabular}

In Table 1, we list the computational time for the Merton jump diffusion model on a 2.5 GHz Quad-Core (but only one core is used) AMD Opteron(tm) Processor. Parameters are fixed to be those in (5.1).

To accurately compute the integral term, we further divide the first subdomain $[0, K]$ into two subdomains $[0,0.1 K]$ and $[0.1 K, K]$. We fix the number of spatial points in $[0,0.1 K]$ to be 4 , and use 60 points to perform over-integration in $[0,0.1 K]$. In terms of the accuracy, our spectral-element method appears to be more efficient than a usual spectral-element method in [40] when comparing Table 1 in this paper with Table 3 in [40], and can be orders of magnitude faster than a low-order finite difference method according to Tables 1 and 2 in [40].

Note that the result in Table 3 of [40] is obtained by using the exact Dirichlet boundary condition at the truncated boundary. However, for most real applications, exact boundary condition is not available and is usually replaced by zero. This could introduce significant irreducible errors. Hence, our method should be more accurate when applied to real problems.

\subsection{Local Volatility Model}

Our scheme can be easily applied to jump diffusion models with local volatility. So as a final example, we compute the price of European put options with a space-time dependent local volatility function under the Merton jump diffusion model. Namely, instead of (3.1), we solve the following PIDE,

$$
v_{\tau}=\frac{1}{2}\left(\sigma^{2} S^{2} v_{S}\right)_{S}+\left[r S-\lambda \kappa S-\left(\sigma^{2} S\right)_{S}\right] v_{S}-(r+\lambda) v+\lambda \int_{0}^{\infty} v(\eta S, \tau) G(\eta) \mathrm{d} \eta,
$$



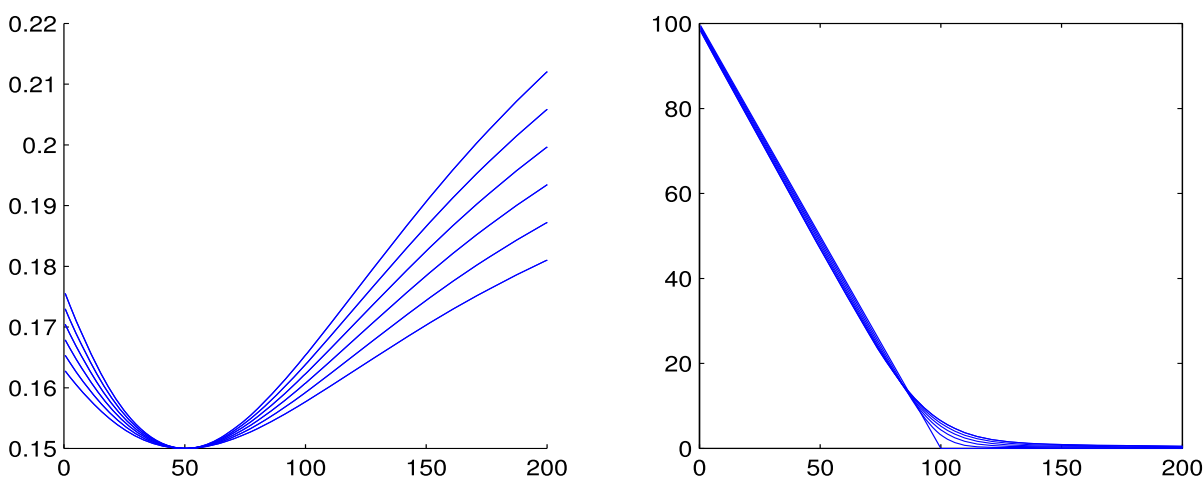

Fig. 11 Left: The local volatility function at $\left\{\tau_{i}\right\}=\{0, T / 5,2 T / 5, \ldots, T\}$ (from bottom to top). Different lines corresponding to different $\sigma(S, \tau)$ as a function of $S$ at different $\tau_{i}$ 's. The horizontal axis is for the variable $S$. The vertical axis is for the value of $\sigma(S, \tau)$. Right: Option prices at $\left\{\tau_{i}\right\}$ (from bottom to top). The horizontal axis is the stock price $S$. The vertical axis is the put option price

where the local volatility function (cf. [35]) is chosen to be

$$
\sigma=\sigma(S, \tau)=0.15+0.15(0.5+2 \tau) \frac{(S / 100-1 / 2)^{2}}{(S / 100)^{2}+1.44} .
$$

Since no exact solution is available, we plot in Fig. 11 the local volatility function at different time steps and the corresponding European put prices. Parameters are chosen as in (5.1) except for $\sigma$, and $\delta t=10^{-3}$.

\section{Conclusions}

We developed an efficient and accurate spectral element scheme for the European option pricing with the PIDE approach. Our method enjoys the following advantages: (i) The unbounded domain is treated directly by using Laguerre functions to avoid the domain truncation error; (ii) the spectral element approach with interior modal basis functions is very efficient and flexible for payoff functions with slope discontinuities and various boundary conditions; (iii) the semi-implicit Crank-Nicholson-Leap-Frog scheme with explicit treatment of integral terms is stable. The numerical results show that our scheme is of secondorder in time and exponentially convergent in space, despite the slope discontinuity. The numerical results also indicate that our method would be more efficient and accurate for real applications where the solution at the right truncated boundary is unknown.

While we have only considered the option pricing with one asset case here, we plan to use some of the techniques developed in this paper, together with the fast spectral sparse grid methods developed recently [33] to deal with price contingent claims with two or more assets.

\section{References}

1. Achdou, Y., Pironneau, O.: Computational Methods for Option Pricing (Frontiers in Applied Mathematics). Frontiers in Applied Mathematics, vol. 30. Society for Industrial and Applied Mathematics, Philadelphia (2005) 
2. Almendral, A., Oosterlee, C.W.: Numerical valuation of options with jumps in the underlying. Appl. Numer. Math. 53(1), 1-18 (2005)

3. Bayraktar, E., Xing, H.: Pricing Asian options for jump diffusion. Math. Finance 21(1), 117-143 (2011)

4. Black, F., Scholes, M.: The pricing of options and corporate liabilities. J. Polit. Econ. 81(3), 637-654 (1973). ArticleType: research-article/Full publication date: May-Jun., 1973/Copyright (C) 1973 The University of Chicago Press

5. Boyd, J.P.: Chebyshev and Fourier Spectral Methods, 2nd edn. Dover, Mineola (2001)

6. Carmona, R., Durrleman, V.: Pricing and hedging spread options. SIAM Rev. 45(4), 627 (2003)

7. Carr, P., Cousot, L.: A PDE-approach to jump-diffusions. Quant. Finance 11(1), 33 (2011)

8. Carr, P., Madan, D.B., Smith, R.H.: Option valuation using the fast Fourier transform. J. Comput. Finance 2, 61-73 (1999)

9. Chen, H., Liu, F., Reich, N., Winter, C., Zhou, A.: Two-Scale finite element discretizaitons for integrodifferential equations. To appear in J. Integral Equ. Appl. (2011)

10. Cont, R.: Frontiers in Quantitative Finance: Volatility and Credit Risk Modeling. Wiley, New York (2008)

11. Cont, R., Voltchkova, E.: A finite difference scheme for option pricing in jump diffusion and exponential Lévy models. SIAM J. Numer. Anal. 43(4), 1596-1626 (2005)

12. de Frutos, J.: A spectral method for bonds. Comput. Oper. Res. 35, 64-75 (2008). ACM ID: 1268230

13. Deville, M.O., Fischer, P.F., Mund, E.H.: High Order Methods for Incompressible Fluid Flow, 1 st edn. Cambridge University Press, Cambridge (2002)

14. d'Halluin, Y., Forsyth, P.A., Vetzal, K.R.: Robust numerical methods for contingent claims under jump diffusion processes. IMA J. Numer. Anal. 25(1), 87-112 (2005)

15. Don, W.S., Gottlieb, D.: The Chebyshev-Legendre method: implementing Legendre methods on Chebyshev points. SIAM J. Numer. Anal. 31, 1519-1534 (1994)

16. Evans, L.C.: Partial Differential Equations, 2nd edn. Am. Math. Soc., Providence (2010)

17. Feng, L., Linetsky, V.: Pricing options in jump-diffusion models: an extrapolation approach. Oper. Res. 56(2), 304-325 (2008)

18. Hurd, T.R., Zhou, Z.: A Fourier transform method for spread option pricing. SIAM J. Financ. Math. 1, 142-157 (2010)

19. Karniadakis, G.E.: Spectral/hp Element Methods for Computational Fluid Dynamics. Oxford University Press, London (2005)

20. Lamberton, D., Lapeyre, B.: Introduction to Stochastic Calculus Applied to Finance, 2nd edn. Chapman, Hall/CRC, London (2007)

21. Lee, R.W.: Option pricing by transform methods: extensions, unification and error control. J. Comput. Finance 7(3), 51-86 (2004)

22. Leentvaar, C.C.W., Oosterlee, C.W.: Multi-asset option pricing using a parallel Fourier-based technique. J. Comput. Finance 12(1), 1-26 (2008)

23. Ma, J., Shen, J., Zhao, Y.: On numerical approximations of forward-backward stochastic differential equations. SIAM J. Numer. Anal. 46, 2636-2661 (2008). ACM ID: 1405082

24. Mayo, A.: Methods for the rapid solution of the pricing PIDEs in exponential and Merton models. J. Comput. Appl. Math. 222(1), 128-143 (2008)

25. Merton, R.C.: Option pricing when underlying stock returns are discontinuous. J. Financ. Econ. 3, 125144 (1976)

26. Pooley, D.M., Vetzal, K.R., Forsyth, P.A.: Convergence remedies for non-smooth payoffs in option pricing. J. Comput. Finance 6, 25-40 (2003)

27. Salmi, S., Toivanen, J.: An iterative method for pricing American options under jump-diffusion models. Appl. Numer. Math. 61, 821-831 (2011)

28. Shen, J.: Efficient spectral-Galerkin method I: direct solvers of second-and fourth-order equations using Legendre polynomials. SIAM J. Sci. Comput. 15(6), 1489-1505 (1994)

29. Shen, J.: Efficient Chebyshev-Legendre Galerkin methods for elliptic problems. In: Ilin, A.V., Scott, R. (eds.) Proceedings of ICOSAHOM'95, pp. 233-240 (1996). Houston J. Math

30. Shen, J., Wang, L.L.: Some recent advances on spectral methods for unbounded domains. Commun. Comput. Phys. 5(2), 195-241 (2009)

31. Shen, J., Tang, T.: Spectral and High-Order Methods with Applications. Mathematics Monograph Series, vol. 3. Science Press, Beijing (2006)

32. Shen, J., Tang, T., Wang, L.-L.: Spectral Methods: Algorithms, Analysis and Applications. Springer Series in Computational Mathematics, vol. 41. Springer, Berlin (2011)

33. Shen, J., Yu, H.: Efficient spectral sparse grid methods and applications to high-dimensional elliptic problems. SIAM J. Sci. Comput. 32(6), 3228 (2010)

34. Tankov, P., Cont, R.: Financial Modelling with Jump Processes, 1st edn. Chapman and Hall/CRC, London (2003) 
35. Toivanen, J.: Numerical valuation of European and American options under Kou's jump-diffusion model. SIAM J. Sci. Comput. 30(4), 1949-1970 (2008)

36. Toivanen, J.: A high-order front-tracking finite difference method for pricing American options under jump-diffusion models. J. Comput. Finance 13(3), 61-79 (2010)

37. Topper, J.: Finite element methods in bond and option pricing. Soc. Comput. Econ. 131 (1999)

38. Zhu, W., Kopriva, D.: A spectral element approximation to price European options. II. The BlackScholes model with two underlying assets. J. Sci. Comput. 39(3), 323-339 (2009)

39. Zhu, W., Kopriva, D.: A spectral element approximation to price European options with one asset and stochastic volatility. J. Sci. Comput. 42(3), 426-446 (2010)

40. Zhu, W., Kopriva, D.A.: A spectral element method to price European options. I. Single asset with and without jump diffusion. J. Sci. Comput. 39(2), 222-243 (2009) 